\title{
Electrophysiological correlates of exemplar-specific processes in implicit and explicit memory
}

\author{
Kristina Küper • Christian Groh-Bordin • \\ Hubert D. Zimmer • Ullrich K. H. Ecker
}

Published online: 27 October 2011

(C) Psychonomic Society, Inc. 2011

\begin{abstract}
The present ERP study investigated the retrieval of task-irrelevant exemplar-specific information under implicit and explicit memory conditions. Subjects completed either an indirect memory test (a natural/artificial judgment) or a direct recognition memory test. Both test groups were presented with new items, identical repetitions, and perceptually different but conceptually similar exemplars of previously seen study objects. Implicit and explicit memory retrieval elicited clearly dissociable ERP components that were differentially affected by exemplar changes from study to test. In the indirect test, identical repetitions, but not different exemplars, elicited a significant ERP repetition priming effect. In contrast, both types of repeated objects gave rise to a reliable old/new effect in the direct test. The results corroborate that implicit and explicit memory fall back on distinct cognitive representation and, more importantly, indicate that these representations differ in the type of stimulus information stored. Implicit retrieval entailed obligatory access to exemplar-specific perceptual information, despite its being task irrelevant. In contrast, explicit retrieval proved to be more flexible with conceptual and perceptual information accessed according to task demands.
\end{abstract}

Keywords Priming · Familiarity · Recollection · Episodic memory $\cdot$ Erp

\footnotetext{
K. Küper $(\bowtie) \cdot$ C. Groh-Bordin • H. D. Zimmer

Brain \& Cognition Unit, Department of Psychology,

Saarland University,

Campus A2 4

66123 Saarbruecken, Germany

e-mail: kristina.kueper@mx.uni-saarland.de

\section{U. K. H. Ecker}

Cognitive Science Laboratories, School of Psychology, University of Western Australia,

Crawley, Australia
}

Cognitive models do not conceptualize memory as a single unitary process but agree that it comprises multiple separate mechanisms, neuronal structures, and/or mental representations. A central distinction is that between explicit and implicit memory. Explicit memory can refer to the recall of items from memory, but also to the episodic recognition of items encountered previously. According to two-process models of recognition memory, it subsumes two distinct memory processes: familiarity and recollection (for a review, see Yonelinas, 2002). Familiarity refers to the unspecific awareness that an item has been encountered before and, as such, does not allow for the retrieval of specific details pertaining to this encounter. Recollection, on the other hand, denotes the conscious and effortful retrieval of both item and contextual detail. In event-related potential (ERP) studies, explicit memory is usually accompanied by more positive-going potentials for correctly identified old items, as compared with correct rejections of new items (for a review, see Rugg \& Allan, 2000). In line with two-process models, this ERP old/new effect can be subdivided into two distinct components (cf. Mecklinger, 2000; Nessler, Mecklinger, \& Penney, 2001; Rugg \& Curran, 2007; Rugg \& Yonelinas, 2003). An early ( $\sim 300-500 \mathrm{~ms})$ mid-frontal old/new effect (FN400) has been related to familiarity mechanisms, whereas a late $(\sim 500-700 \mathrm{~ms})$ parietal old/new effect (the late positive complex, LPC) has been related to recollection processes.

Implicit memory, on the other hand, is thought to be independent of conscious awareness and is best explained as a processing advantage for previously encountered stimuli: Responses to previously seen items are faster and less error prone than those to new items (for reviews, see Schacter \& Buckner, 1998; Tulving \& Schacter, 1990). The ERP correlate of this repetition priming effect is a centroparietally focused positivity between 300 and $700 \mathrm{~ms}$ 
poststimulus that is more pronounced for repeated than for new items (Rugg et al., 1998; for a review, see Paller, 2001). According to Schendan and Kutas $(2003,2007)$, this effect can be subdivided into an earlier fronto-central component (N350) indexing initial object identification and implicit memory and a later posterior component (N400/P600) linked to secondary categorization processes and, possibly, incidental explicit recollection. In addition, more pronounced positivities for repeated items have sometimes been reported around $200 \mathrm{~ms}$ poststimulus (Guillaume et al., 2009; Henson, Rylands, Ross, Vuilleumier, \& Rugg, 2004; Schendan \& Kutas, 2007).

It has been suggested that implicit and explicit memory rely on distinct cognitive representations, which themselves are mediated by separable neural systems (Schacter, 1994; Squire, 1994; see also Zimmer \& Ecker, 2010). Support for this assumption comes from research indicating significant differences in the perceptual specificity of implicit and explicit memory for visual objects. Implicit memory has long been considered hyperspecific, in that repetition priming effects are contingent on the overlap of perceptual study and test information (for reviews, see Roediger \& McDermott, 1993; Roediger \& Srinivas, 1993). For instance, behavioral priming effects are significantly reduced when an item is presented in different modalities at study and test (Rajaram \& Roediger, 1993). Similarly, reduced priming effects have been reported for format changes (Weldon, Roediger, Beitel, \& Johnston, 1995) and changes to the shape or outline of an item-for example, when different exemplars of an object are presented at study and test (Biederman \& Cooper, 1992; Cave, Bost, \& Cobb, 1996). Perceptual priming effects are, however, unaffected by study-test changes to arbitrary stimulus features that do not substantially contribute to object identification, such as color, size, or orientation (Biederman \& Cooper, 1992; Zimmer, 1995). In contrast, despite generally being considered the more conceptual process, explicit recognition memory is reduced by both exemplar changes and changes to arbitrary stimulus features (Cave et al., 1996; Cooper, Schacter, Ballesteros, \& Moore, 1992; Zimmer, 1995).

The type-token model by Zimmer and Ecker (2010) offers a theoretical framework that can accommodate these differences in perceptual specificity. According to this model, object memory falls back on three distinct types of representations: type traces, object tokens, and episodic tokens. First, implicit memory results from access to type traces, basic sensory representations that code the diagnostic features of objects - that is, features that are crucial for object identification, such as outline and shape. Repetition priming effects are thus diminished only when diagnostic item features are changed between study and test but are unaffected by changes of arbitrary perceptual features (for similar considerations, see Srinivas, 1996).
Second, familiarity results from access to integrated memory representations. According to Treisman's feature integration theory, visual object features, such as color, shape, and size, are first processed separately in a distributed network, before they are bound into a coherent representation, the object file (Treisman, 2006; Treisman \& Gelade, 1980). Zimmer and Ecker (2010) proposed that when an exemplar disappears from view, the corresponding object file is not lost but persists as a memory representation they labeled object token. Access to these object tokens engenders the feeling of familiarity. Since object tokens integrate all perceptual features pertinent to a specific exemplar, familiarity should be affected by any perceptual manipulation.

Finally, the type-token model postulates that an integrated representation of the object token and its encoding context is stored as a so-called episodic token that is thought to support conscious recollection. Recollection is considered a highly controlled process that can be adapted to task demands. It therefore requires flexible and controlled access to such a higher-order representation. It follows that the emergence of visual specificity effects on measures of recollection is contingent on task demands.

As described above, ERPs have been instrumental in dissociating implicit and explicit memory processes. In the same vein, they can also help determine whether these memory processes access distinct mental representations that differ in the type and detail of information stored. Concerning recollection, a number of ERP studies support the type-token model in that the correlate of recollection, the LPC, is reliably diminished by task-relevant changes to the context of study items (Ecker, Zimmer, \& Groh-Bordin, $2007 \mathrm{a}, \mathrm{b})$. The picture is somewhat less clear when it comes to familiarity and perceptual priming, however.

Changes to perceptual intraitem features have reduced the FN400 old/new effect associated with familiarity in some cases (Ecker et al., 2007a, b; Groh-Bordin, Zimmer, \& Ecker, 2006; Schloerscheidt \& Rugg, 2004), but not in others (Curran, 2000; Curran \& Cleary, 2003; Curran \& Dien, 2003). It is feasible that these conflicting result patterns have emerged as a result of differences in task demands - in particular, perceptual processing demands (cf. Ecker, Arend, Bergström, \& Zimmer, 2009). In the past, perceptual specificity effects on the FN400 have been reported almost exclusively under conditions that encouraged perceptual processing, be it by presenting difficult-to-identify stimuli (Groh-Bordin, Zimmer, \& Mecklinger, 2005), by instructing subjects to encode the objects together with their perceptual features (e.g., Ecker et al., 2007a), or by inducing a perceptually specific retrieval orientation (Ecker \& Zimmer, 2009).

ERP correlates of implicit memory, on the other hand, appear to be unaffected by changes to arbitrary perceptual features. Equivalent ERP repetition priming effects have been found for identically repeated objects and their mirror reversals 
(Groh-Bordin et al., 2005). Similarly, Schendan and Kutas (2007) presented subjects with identical versus complementary object fragments at study and test (i.e., fragments portrayed the same object, but with perceptually identical vs. nonoverlapping fragments, respectively). They identified an ERP repetition effect (N350) that indexed the reactivation of global shapes but was insensitive to the specific test fragment used. It would be premature to interpret these results in favor of the type-token model, however, since they could reflect simple null results, in that the ERP correlates of implicit memory are never affected by perceptual manipulations, regardless of whether the changed feature is arbitrary or diagnostic - that is, pertaining to the shape of the visual object. To our knowledge, as of yet, only one ERP study has investigated study-test changes to diagnostic object features. Schendan and Kutas (2003) reported ERP repetition priming effects that exhibited some viewpoint specificity: Both the N350 and a subsequent N400/P600 were more positive-going for identically repeated items than for objects presented from a different view at test than at study. Yet these format effects appeared limited to unusual test views that likely required increased perceptual processing and did not emerge when test views were canonical.

In the context of perceptual specificity of memory in general, and the type-token model in particular, two central issues are thus as yet unresolved: (1) Is enhanced perceptual stimulus processing a prerequisite for the emergence of perceptual specificity effects on ERP correlates of familiarity? (2) Are ERP correlates of implicit memory reliably modulated by study-test changes to the shape of visual objects? The main goal of the present study was to address both of these issues by examining the impact of exemplar changes from study to test on the ERP correlates of repetition priming and recognition memory, under conditions that discouraged enhanced perceptual processing.

To this end, two subject groups incidentally studied pictures of objects by performing a natural/artificial discrimination task. In an effort to minimize perceptual processing, we employed easily identifiable photographs of real-life objects as stimuli. At test, one subject group again attended the semantic discrimination task (implicit group), while the other group performed a recognition memory task (explicit group). In both groups, there were three types of test stimuli: new objects, identical repetitions of studied items, and different exemplars of studied objects. Different test exemplars were thus conceptually similar to a studied item but differed in a number of diagnostic perceptual features coding the identity of a specific exemplar. The direct recognition test was an inclusion task in which both identical repetitions and different exemplars had to be accepted as old.

Since the direct task demands thus put little emphasis on perceptual processing, we expected the parietal ERP old/new effect (LPC) associated with recollection to be independent of perceptual study-test congruency and, thus, similarly pronounced for both kinds of old items. The same rationale applies to familiarity and the midfrontal component (FN400). The reduced need for perceptual processing and the conceptually focused test task should reduce the perceptual specificity found in other studies with stronger perceptual processing demands (cf. Ecker \& Zimmer, 2009). In contrast, diminished FN400 old/new effects for different exemplars would attest to the retrieval of exemplar-specific information in the course of familiarity even in situations with low perceptual processing demands.

On the premise that implicit memory representations code diagnostic shape information, we expected the ERP repetition priming effect for different exemplars to be diminished or even absent in the indirect test. We thus anticipated clearly dissociable specificity effects for the two test groups. As such, the present results potentially not only could resolve open issues with the type-token model, but also could provide further insight into differences in cognitive and neural representations associated with implicit and explicit memory.

\section{Method}

\section{Subjects}

The implicit and the explicit groups each comprised 24 right-handed students of Saarland University. Subjects had normal or corrected-to-normal vision and were paid for their participation. In the explicit group, 2 subjects had to be excluded from further analyses: One did not follow instructions properly; the other showed excessive EEG artifacts (leaving fewer than 15 trials per condition for averaging). In the implicit group, 1 subject was excluded because of excessive EEG artifacts. Hence, for behavioral and ERP analysis, 22 subjects remained in the explicit group (mean age, 26.9 years; range, 19-51; 10 female), while 23 remained in the implicit group (mean age, 26.5 years; range, $21-38$; 9 female).

\section{Stimuli}

Stimuli were 134 photographs of real objects, with one half depicting natural and the other half artificial objects (see examples in Fig. 1). Stimuli were presented in the center of a 17-in. color monitor with a resolution of 1,024 $\times 768 \mathrm{dpi}$ against a white background. Items subtended a visual angle of approximately $3^{\circ}$, since their longer side spanned 250 pixels. Assignment of stimuli to experimental conditions (see below) was counterbalanced across subjects. 
Fig. 1 Examples of artificial (left half) and natural (right half) objects used in the present study (top row), as well as their corresponding different exemplars (bottom row)
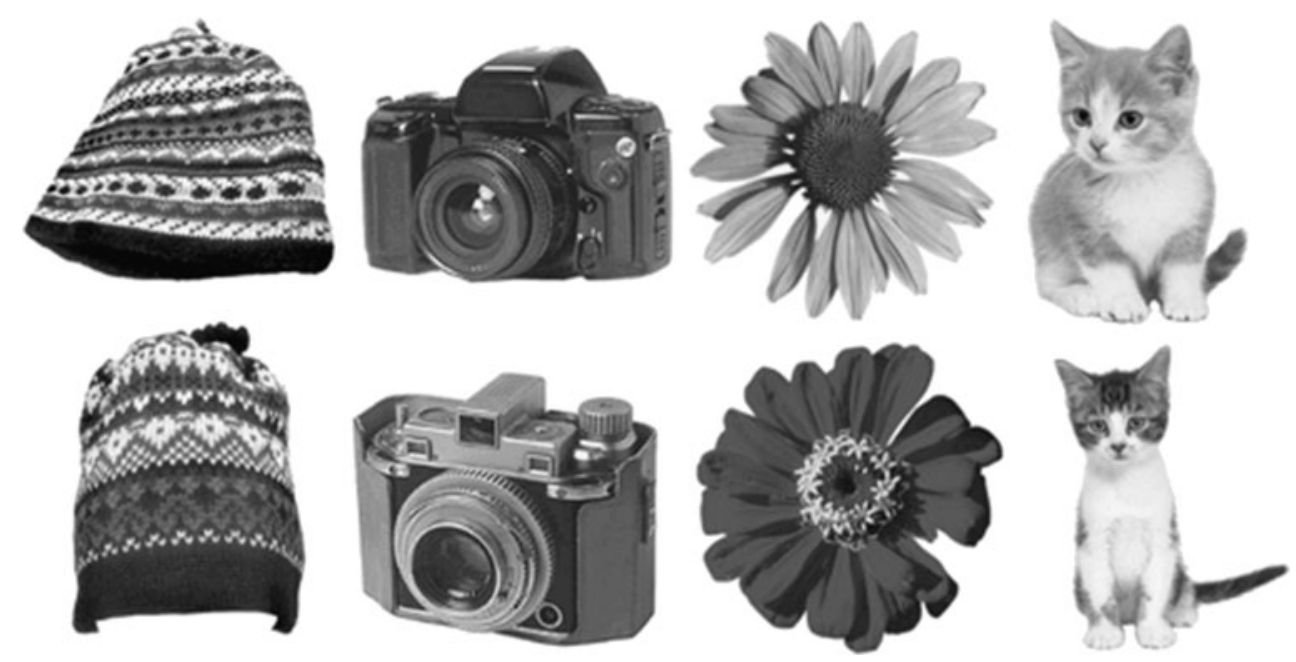

Design and procedure

During study, both groups incidentally learned 64 objects by judging whether each photo depicted a natural or an artificial object. ${ }^{1}$ At the beginning of each trial, a fixation cross was presented for $500 \mathrm{~ms}$, followed by a blank screen $(500 \mathrm{~ms})$. Afterward, the stimulus was presented for $1,500 \mathrm{~ms}$, and subjects made a natural/artificial response within $2,000 \mathrm{~ms}$ by pressing the shift-left or shift-right key with one of their index fingers (assignment of response keys was counterbalanced across subjects). The intertrial interval was $1,000 \mathrm{~ms}$.

At test, both groups were presented with 128 objects, 64 of which had been previously seen during study and 64 that were new. For half of the repeated objects, the same photograph was presented again (old same); for the other half, a photograph depicting a different exemplar of the respective object category was shown (old different; see examples in Fig. 1). Subjects from the implicit group had to perform the natural/artificial judgment task again, whereas subjects from the explicit group were given an unexpected recognition memory test. The latter test was an inclusion task; subjects were informed about the occurrence of different exemplars and were told to accept both old same and old different objects as old. Trial timing was identical to that in the study phase.

\section{EEG recording and analyses}

The experiment was run in a sound- and electromagnetically shielded chamber. EEG activity was recorded contin-

\footnotetext{
${ }^{1}$ Six additional items were used in a short practice block prior to the study phase in order to familiarize subjects with the procedure and the trial timing.
}

uously from $64 \mathrm{Ag} / \mathrm{AgCl}$ electrodes mounted in a preconfigured cap (Easy Cap, Easycap GmbH, Herrsching-Breitbrunn, Germany), arranged according to the international 10-10 system. Impedances for all electrodes were kept below $10 \mathrm{k} \Omega$. Signals were digitized with a sampling rate of $250 \mathrm{~Hz}(70 \mathrm{~Hz}$ low-pass, $50 \mathrm{~Hz}$ notch filter) using an $\mathrm{AC}$ coupled amplifier (Brain Amp MR, Brain Products $\mathrm{GmbH}$, Munich; time constant, $10 \mathrm{~s}$ ) and were referenced online to the left mastoid electrode. For further analysis, electrodes were rereferenced offline to averaged mastoids. Vertical and horizontal ocular artifacts were monitored and corrected offline (Gratton, Coles, \& Donchin, 1983).

ERPs were extracted during the test phase, from -200 to $1,000 \mathrm{~ms}$ around stimulus onset. Data were baseline corrected with respect to the $200 \mathrm{~ms}$ prestimulus interval and digitally band-pass filtered at $0.2-20 \mathrm{~Hz}$ (slope, $24 \mathrm{~dB}$ ). Trials with incorrect responses and epochs containing artifacts (maximum amplitude in the recording epoch, $\pm 150 \mu \mathrm{V}$; maximum difference between two successive sampling points, $50 \mu \mathrm{V}$; maximum difference of two values in the interval, $150 \mu \mathrm{V}$; lowest allowed activity change, $0.5 \mu \mathrm{V}$ in successive intervals of $100 \mathrm{~ms}$ ) were excluded from averaging.

For both groups, ERPs were computed for the three different conditions (as was stated above, a minimum of 15 trials per condition was considered necessary for inclusion into the grand average; the mean numbers of valid trials per condition are given in parentheses in the following): new (implicit 58/explicit 49), old same (29/27), old different $(29 / 23)$. To simplify statistical analysis of ERPs, the following nine regions of interest (ROIs) were built: leftfrontal, F5, F7, FC5; left-central, T7, CP5, TP7; leftparietal, P5, P7, PO7; mid-frontal, Fz, FC1, FC2; midcentral, Cz, CP1, CP2; mid-occipito-parietal, Pz, O1, O2; 
and the right counterparts of left-sided electrode regions. This procedure resulted in a 3 (anterior/posterior) $\times 3$ (laterality) electrode arrangement that was used in the statistical analyses (see below). Early (300 to $500 \mathrm{~ms}$ ) and late $(500$ to $700 \mathrm{~ms})$ time windows were selected for analysis to cover ERP correlates of both implicit and explicit memory (cf. Nessler et al., 2001; Schendan \& Kutas, 2007). EEG data were thus analyzed in 3 (anterior/ posterior) $\times 3$ (laterality) $\times 3$ (condition) ANOVAs for each task and time window. Subsidiary analyses are reported for the highest order main effect or interaction that reached significance.

\section{Results}

\section{Behavioral data}

During study, performance in the artificial/natural judgment task was comparable in both experimental groups with respect to accuracy (implicit group, 97\% correct responses; explicit group, $96 \%$ correct), $F(1,43)=2.83, p=.10$, and reaction times (RTs; implicit, $644 \mathrm{~ms}$; explicit, $680 \mathrm{~ms}$ ), $F<1$.

For each subject group (implicit vs. explicit), mean accuracy and RTs for the test phase (see Table 1) were submitted to a separate ANOVA with the within-subjects factor of condition (new, old same, old different) .

Implicit group $\mathrm{RT}$ analysis revealed a significant main effect of condition, $F(1,22)=10.08, p<.001$. According to follow-up analyses, subjects responded more quickly to old items than to new ones, $F(1,22)=12.85, p<.001$ (single comparisons, same-new, different-new, both $p$ s $<.01$ ). RTs to old same and old different items differed marginally from each other, $F(1,22)=2.65, p=.12$. The corresponding effect size was small $\left(\eta_{\mathrm{p}}{ }^{2}=.11\right.$ ), and power analyses (using G*Power3; Faul, Erdfelder, Lang, \& Buchner, 2007) indicated that the present experiment did not have sufficient power to detect an effect of such small size $(1-\beta=.32)$. With respect to accuracy, items in the three conditions did not differ significantly from each other, $F(1,22)<1$.
Explicit group RTs showed a significant main effect of condition, $F(1,21)=26.85, p<.001$. Planned comparisons revealed that subjects responded faster to old items than to new ones, $F(1,21)=25.48, p<.001$ (single comparisons, same-new, different-new, both $p$ s $<.01$ ) and faster to same stimuli than to different ones, $F(1,21)=31.79, p<.001$. The accuracy data also yielded a significant main effect of condition, $F(1,21)=11.79, p<.001$, that was due to higher accuracy for old same items than for both old different, $F(1$, $21)=38.27, p<.001$, and new, $F(1,21)=6.97, p<.01$, items. An analysis of $\operatorname{Pr}$ discrimination scores (to proportion of hits-proportion of false alarms; Snodgrass \& Corwin, 1988) yielded comparable results: Performance was above chance for both old same, $t(21)=21.41, p<$ .001 , and old different, $t(21)=10.69, p<.001$, items, and significantly better in the same $(\operatorname{Pr}=.75)$ than in the different $(\operatorname{Pr}=.48)$ condition, $F(1,21)=38.19, p<.001$.

\section{ERP data}

The EEG data were analyzed in 3 (anterior/posterior) $\times 3$ (laterality) $\times 3$ (condition) ANOVAs for each task and time window.

\section{Implicit group}

As compared with new items, identically repeated objects, but not different exemplars, elicited a widespread positive deflection beginning as early as about $300 \mathrm{~ms}$ poststimulus-onset (see Fig. 2).

300-500 $\mathrm{ms}$ Significant main effects and interactions are listed in Table 2 (results were Greenhouse-Geisser corrected where appropriate). Subsequent condition analyses at each ROI were all significant, $F \mathrm{~s}(2,44)$ between 5.09 and 18.79 , all $p \mathrm{~s}<.05$. Follow-up contrasts indicated that at all but the left anterior ROI, these condition effects were due to old same items differing significantly from both new items, $F_{\mathrm{s}}(1,22)$ between 7.56 and 41.2 , all $p \mathrm{~s}<.05$, and old different items, $F \mathrm{~s}(1,22)$ between 6.02 and 17.66, all $p \mathrm{~s}<$

Table 1 Mean reaction times (RTs) and accuracy in the test phase as a function of memory task group (implicit, explicit) and condition (new, old same, old different)

\begin{tabular}{|c|c|c|c|c|c|c|}
\hline & \multicolumn{2}{|l|}{ New } & \multicolumn{2}{|l|}{ Old Same } & \multicolumn{2}{|l|}{ Old Different } \\
\hline & Percent Correct & RT & Percent Correct & RT & Percent Correct & RT \\
\hline Implicit group & $97(1)$ & $625(31)$ & $97(1)$ & $587(24)$ & $97(1)$ & $597(28)$ \\
\hline Explicit group & $80(2)$ & $1001(32)$ & $87(2)$ & $825(25)$ & $74(2)$ & 914 (29) \\
\hline
\end{tabular}

Note. Percent correct denotes the percentage of correct responses; RT indicates reaction time; values in parentheses display the standard errors of the means 

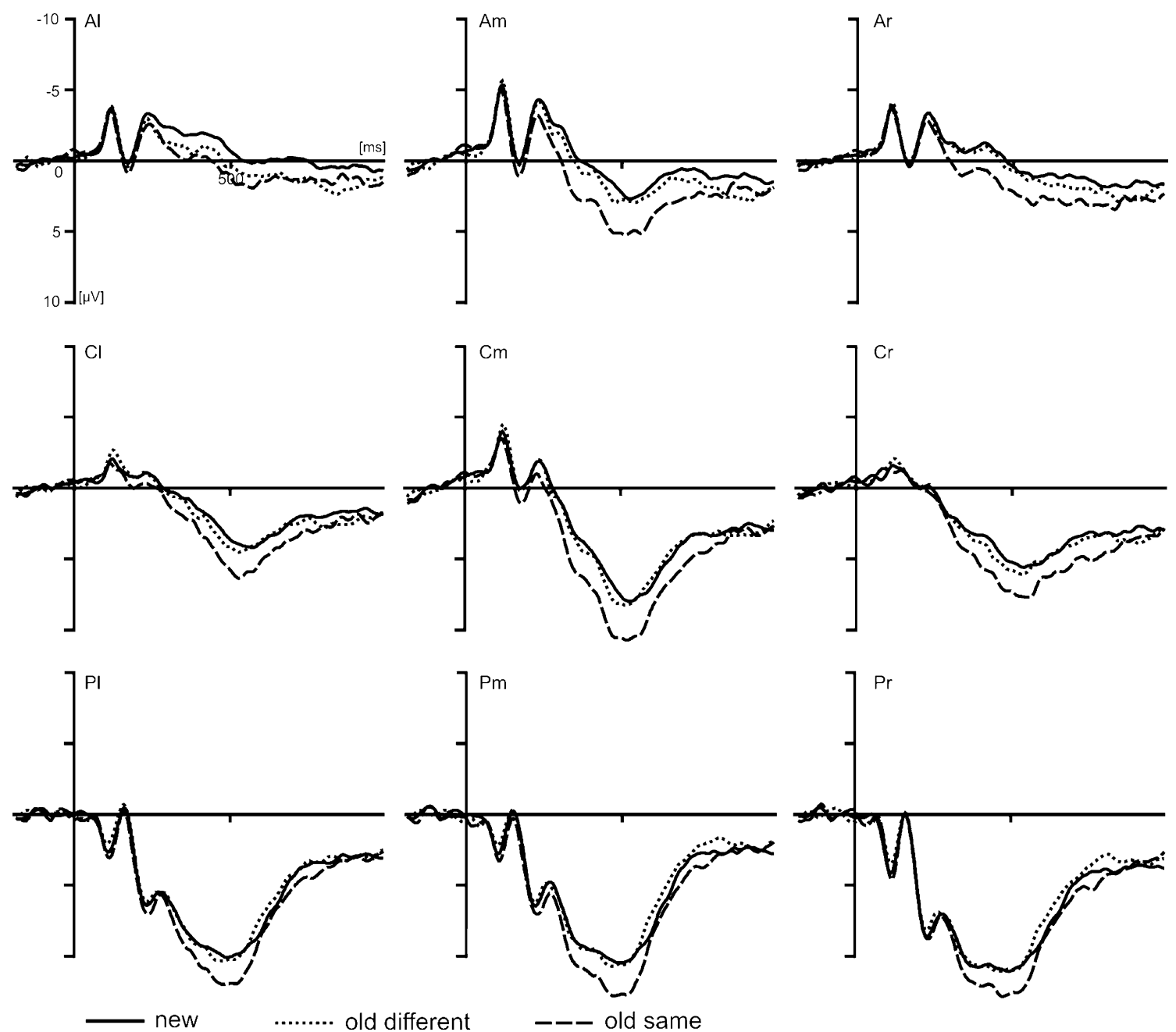

Fig. 2 Grand average waveforms at nine regions of interest in the implicit group as a function of condition (new, old same, old different). A, anterior; $\mathrm{C}$, central; $\mathrm{P}$, posterior; 1 , left; $\mathrm{m}$, middle, $\mathrm{r}$,

right. Time scaling ranges from -200 to $1,000 \mathrm{~ms}$ around stimulus onset. Positive deflections are displayed downward

.05 , while old different and new items elicited similar ERPs, $F \mathrm{~s}(1,22)$ between 0.01 and 1.75 , all $p \mathrm{~s}>.23$. At the left anterior ROI, however, old different items differed from new items, $F(1,22)=8.13, p<.01$, but not from identical repetitions, $F(1,22)=1.75, p=.2$.

500-700 ms Follow-up analyses at anterior, central, and posterior ROIs all gave rise to significant main effects of condition, $F_{\mathrm{s}}(2,44)$ between 7.01 and 11.99 , all $p \mathrm{~s}<.01$, as did follow-ups at left, middle, and right ROIs, $F \mathrm{~s}(2,44)$ between 7.09 and 13.19, all $p$ s $<.01$. Across all subsidiary analyses, old different items elicited similar ERPs as new items, $F_{s}(1,22)$ between $<1$ and 2.32 , all $p s>.14$. In contrast, old same items gave rise to more positive-going ERPs than did both old different items, $F_{\mathrm{s}}(1,22)$ between 7.07 and 23.05 , all $p \mathrm{~s}<.05$, and new items, $F_{\mathrm{s}}(1,22)$ between 10.01 and 30 , all $p \mathrm{~s}<.01$, in all subsidiaries, except for the posterior ROI, where the divergence from new items only approached significance, $F(1,22)=3.97, p=.059$. Visual inspection of the ERP waveforms suggested a divergent result pattern in the left anterior ROI as the cause of the significant condition $\times$ laterality interaction. At this ROI, as in the earlier time interval, old different items appeared to differ from new items, but not from identical repetitions.

\section{Explicit group}

Across all ROIs, old items elicited more positive-going waveforms than did new ones from around $300 \mathrm{~ms}$ poststimulus-onset until the end of the recording epoch (see Fig. 3).

300-500 ms Significant main effects and interactions are listed in Table 2 (results were Greenhouse-Geisser corrected where appropriate). Main effects of condition proved stable at anterior, central, and posterior ROIs, $F \mathrm{~s}(2,42)$ between 5.7 and 12.93, all $p \mathrm{~s}<.01$. Post hoc comparisons 
Table 2 Results of the analyses of variance of ERP effects as a function of memory task group (implicit, explicit) and time interval $(300-500 \mathrm{~ms}$, 500-700 ms)

\begin{tabular}{llllll}
\hline Memory Type & Time Interval & Effect & $F$ & $p$ & $\varepsilon$ \\
\hline \multirow{2}{*}{ Implicit } & \multirow{2}{*}{$300-500 \mathrm{~ms}$} & Cond & 11.99 & $<.001$ & .9 \\
& & Cond $\times$ AP & 3.78 & $<.05$ & .62 \\
& & Cond $\times$ Lat & 4.71 & $<.01$ & .72 \\
& & Cond $\times$ AP $\times$ Lat & 2.43 & $<.05$ & .54 \\
& $500-700 \mathrm{~ms}$ & Cond & 10.62 & $<.001$ & .94 \\
& & Cond $\times$ AP & 5.94 & $<.01$ & .55 \\
& & Cond $\times$ Lat & 3.12 & $<.05$ & .65 \\
& & Cond $\times$ AP $\times$ Lat & 1.55 & n.s. & .69 \\
& & Cond & 11.19 & $<.001$ & .88 \\
& $300-500 \mathrm{~ms}$ Exlicit & Cond $\times$ AP & 5.44 & $<.01$ & .65 \\
& & Cond $\times$ Lat & 1.29 & n.s. & .93 \\
& & Cond $\times$ AP $\times$ Lat & $<1$ & n.s. & .71 \\
& & Cond & 17.82 & $<.001$ & .93 \\
& & Cond $\times$ AP & $<1$ & n.s. & .57 \\
& & Cond $\times$ Lat & 1.23 & n.s. & .86 \\
& & Cond $\times$ AP $\times$ Lat & $<1$ & n.s. & .64 \\
\hline
\end{tabular}

Note. $\varepsilon$ denotes GreenhouseGeisser epsilon values; Cond denotes condition; AP stands for anterior/posterior, and Lat for laterality. See the text for further explanation scaled difference waves, $F(2,86)=8.88, p<.001$ (cf. McCarthy \& Wood, 1985; Urbach \& Kutas, 2002; Wilding, 2006). Post hoc analyses indicated that differences between implicit and explicit remembering emerged between 500 and $700 \mathrm{~ms}$ at central, $F(1,43)=5.07, p<.05$, and posterior, $F(1,43)=6.3, p<.05$, ROIs (all other $p \mathrm{~s}>.2$ ).

These post hoc analyses also highlighted differences in the topographies of the explicit old/new effects in the early and late time windows: Retrieval-related positivities at central, $F(1,43)=12.55, p<.001$, and posterior, $F$ $(1,43)=10.33, p<.01$, ROIs were more pronounced in the late than in the early time window [anterior ROI, $F(1$, $43)=1.64, p=.2$. In contrast, topographical differences across time windows were not observed for the implicit group (all $p \mathrm{~s}>.3$ ).

\section{Discussion}

The present study examined the effects of exemplar changes from study to test on ERP correlates of implicit and explicit memory. To this end, two subject groups were presented with pictures of objects in an incidental study phase. At test, subjects had to perform either a semantic judgment task (indirect test) or a recognition memory task (direct test) on new items, identical versions, and different exemplars of the previously studied items.

Explicit and implicit remembering elicited different and clearly separable ERP correlates, as would be expected if both types of memory constituted separate neural processes based on distinct cognitive representations. More important, 

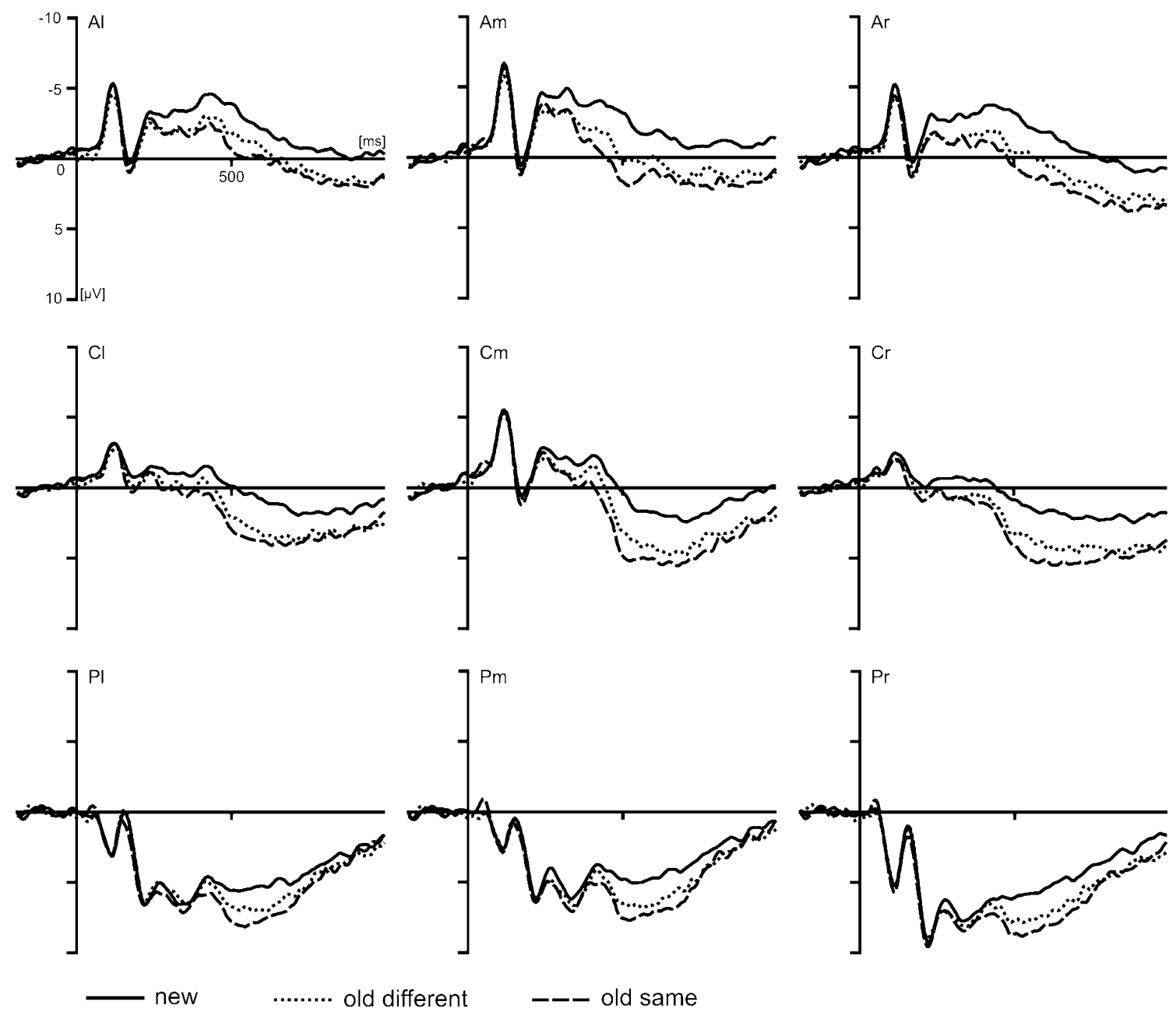

........ old different

Fig. 3 Grand average waveforms at nine regions of interest in the explicit group as a function of condition (new, old same, old different). A, anterior; C, central; P, posterior; 1, left; $\mathrm{m}$, middle; r, right. Time

behavioral and ERP correlates of implicit and explicit memory were differentially affected by perceptual studytest congruency. In the implicit group, ERP priming effects were confined to identical repetitions: As compared with new items, only old same, but not old different, items gave rise to a pronounced centro-parietal positivity between 300 and $700 \mathrm{~ms}$ poststimulus. Behavioral priming effects were generally small and limited to RTs, since performance was at ceiling with respect to accuracy. Still, RT priming effects emerged for both types of old items but were marginally more pronounced for old same than for old different items. We thus observed repetition effects for different exemplars in behavioral, but not in ERP, measures. Such dissociations between behavioral and ERP priming effects are not unusual (e.g., Schendan \& Kutas, 2007) and are most likely due to the fact that ERP correlates reflect single processing stages, whereas RTs reflect the cumulative effect of these processing stages (cf. Vogel, Luck, \& Shapiro,
1998). Given that subjects in the present study had to perform identical tasks at study and indirect test, it is additionally possible that RTs were substantially modulated by response-related processes, such as response priming (Horner \& Henson, 2009), that have only a negligible effect on the ERP correlates of implicit item memory (cf. Race, Badre, \& Wagner, 2010; Schacter, Dobbins, \& Schnyer, 2004).

In the explicit group, both old same and old different items elicited stable ERP old/new effects of similar magnitude, with the LPC appearing marginally more pronounced for same than for different exemplars. Similarly, RT and accuracy old/new effects emerged for both types of old items but were significantly more pronounced for identical repetitions than for different exemplars. Performance measures were thus unambiguously affected by study-test congruency, whereas the perceptual mismatch was not sufficient to produce a reliable congruence effect in ERPs. In line with 


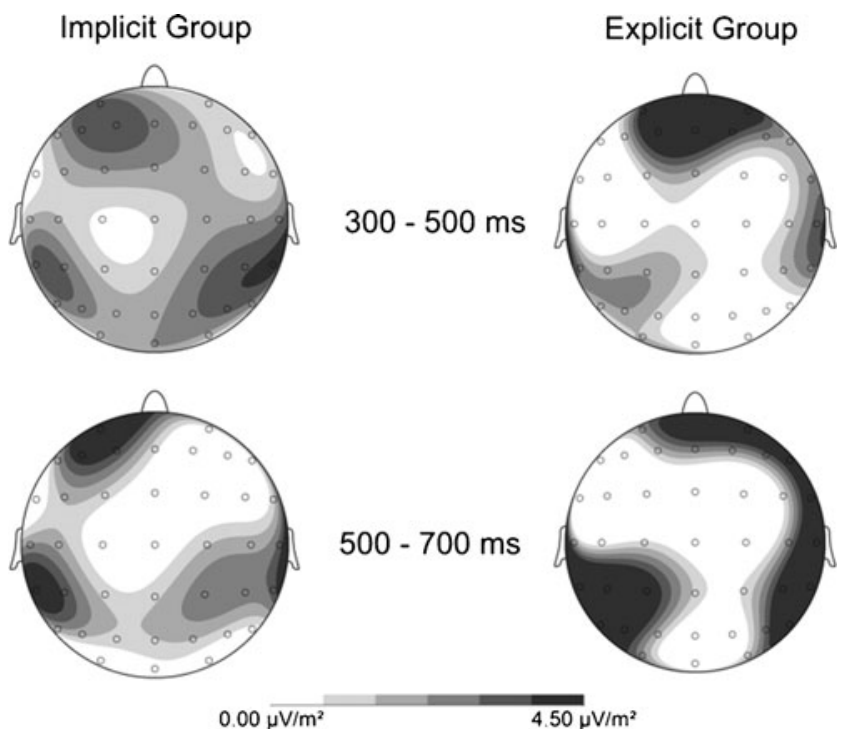

Fig. 4 Spherical-spline interpolated topographical maps of ERP old/ new differences in the 300 to $500 \mathrm{~ms}$ and 500 to $700 \mathrm{~ms}$ time intervals as a function of memory task group (implicit, explicit). ERPs to new items were subtracted from old same items. The head is depicted from above with the front facing up, left on the left, and so forth

two-process models of recognition memory, behavioral performance has been linked to the LPC, rather than the FN400 (Curran \& Cleary, 2003). Still, dissociations between behavioral old/new effects and LPC modulation have been observed frequently, particularly in inclusion memory tasks (e.g., Groh-Bordin et al., 2005; Tsivilis, Otten, \& Rugg, 2001). Ecker et al. (2007a) even reported higher accuracy and shorter RTs for new than for studied items, indicating that behavioral performance in inclusion tasks can be strongly modulated by factors other than explicit memory. This is hardly surprising, since the inclusion task requires subjects to categorize new items as "old" when they are conceptually similar to study items. Hence, conflict detection and resolution mechanisms, as well as corresponding decision processes, probably come to play in the categorization of different exemplars (Ecker \& Zimmer, 2009). Given the cumulative nature of behavioral measures, such processes will more likely affect RT and accuracy measures than they will affect specific ERP correlates of explicit memory (cf. Vogel et al., 1998).

In our discussion of the data, we will thus focus on ERP correlates of implicit and explicit memory, rather than on behavioral performance, since the former provide a more process-pure estimate of memory effects.

With respect to the implicit group, the present data are the first to demonstrate that ERP repetition priming effects can be modulated by exemplar changes from study to test. It has already been established that changes to accidental stimulus features do not reduce ERP priming effects (Groh-Bordin et al., 2005; Schendan \& Kutas, 2007). The present results dispel the notion that ERP correlates of implicit memory are thus generally unsusceptible to perceptual congruency manipulations. As we have suggested earlier (Groh-Bordin et al., 2005), it instead appears that only changes to diagnostic features that affect the identification of a specific stimulus reduce the repetition effect. In this respect, the present data are in line with the type-token model (Zimmer \& Ecker, 2010), which posits that perceptual repetition priming results from temporary changes to modality-specific sensory representations, labeled type traces.

According to the model, type traces code only diagnostic sensory features necessary for stimulus identification, such as object shape and outline. In contrast, accidental features irrelevant for identification are not included in type traces. This raises the question as to how to define diagnostic stimulus features. Schendan and Kutas (2003, 2007) demonstrated that viewpoint changes from canonical to unusual views reduced ERP repetition priming effects, whereas presenting different object fragments at study and at test did not. In addition, behavioral priming effects have been found to be unaffected by changes to stimulus color, size, or orientation (e.g., Biederman \& Cooper, 1992; Zimmer, 1995). It thus appears that global shape information, but not local stimulus details, constitutes diagnostic features that are included in type traces. As such, type traces would be conceptually similar to the object-centered 3-D representations at the core of view-invariant models of object identification (e.g., Marr, 1982). The present data, however, demonstrate that type traces must additionally contain exemplar-specific information that allows for the identification of single object instances. We found reliable ERP repetition priming effects only for identical repetitions, but not for different exemplars of the same object category. It thus appears that the representation mediating repetition priming effects is a perceptual one that codes for the form information of single object instances.

There is some debate on whether indirect memory tasks such as the semantic judgment task used in the present study exclusively tap into implicit memory processes. It is instead feasible that during the course of an indirect memory task, incidental explicit retrieval of the test stimulus will occur on at least a small number of trials (Schacter, Bowers, \& Booker, 1989). Whereas the earlier portion (N350) of the ERP repetition effect is considered a relatively pure index of implicit memory, its later portion (N400/P600) has previously been linked to such involuntary explicit retrieval (Guillaume et al., 2009; Schendan \& Kutas, 2003, 2007). For the present study, it seems unlikely, however, that the ERP repetition effect observed between 500 and $700 \mathrm{~ms}$ reflects explicit, rather than implicit, retrieval. In the later time window, ERP correlates of implicit and explicit memory as exhibited by our two test 
groups were clearly dissociable not only in terms of perceptual specificity, but also with respect to their topographical distribution. ${ }^{2}$

Concerning the explicit group, we observed reliable perceptual specificity effects in behavioral, but not in ERP, measures. Most notably, same and different exemplars elicited a comparable FN400 old/new effect that has previously been linked to familiarity mechanisms (e.g., Rugg \& Curran, 2007). These ERP results are in line with previous research by Curran and colleagues (Curran, 2000; Curran \& Cleary, 2003; Curran \& Dien, 2003) but conflict with a large number of studies in which the FN400 old/new effect was, instead, diminished by perceptual study-test manipulations (Ecker et al., 2007a, b; Groh-Bordin et al., 2006; Groh-Bordin et al., 2005; Grove \& Wilding, 2009; Schloerscheidt \& Rugg, 2004). Again, we can draw on the type-token model to explain these contrasting results.

According to the model, the representation subserving familiarity, the object token, binds diagnostic and accidental attributes of previously encountered objects. As a result, both perceptual and conceptual information processing contribute to familiarity-based recognition to a varying degree (for similar considerations, see Curran \& Dien, 2003, and Ecker \& Zimmer, 2009). The degree to which these different types of information processing are utilized in a given task may depend on task characteristics and the specific properties of the stimuli used. A number of studies from our lab have reported perceptual specificity effects on the FN400. All of these studies put emphasis on perceptual encoding, either by way of instruction or because of the stimuli used. For example, in Groh-Bordin et al. (2005), the mid-frontal old/new effect was eliminated for items that were perceptually modified from study to test. As opposed to the present study, however, the pictorial stimuli employed in this earlier study were extremely difficult to identify and thus required enhanced perceptual processing, which in turn may have inflated the perceptual specificity of the familiarity component. A recent study by Ecker and colleagues indicated that perceptual processing at study may be a prerequisite for the emergence of perceptual specificity effects on the FN400 (Ecker et al., 2009). The authors found that perceptual study-test changes did not

\footnotetext{
${ }^{2}$ Rugg and colleagues (Rugg et al., 1998) were able to identify an ERP index of implicit retrieval in a direct recognition memory task: A posterior positivity between 300 and $500 \mathrm{~ms}$ was more pronounced for studied items that were not recognized, as compared with correct rejections of new items. A comparison of ERPs for misses in the direct task and hits in the indirect task could thus potentially resolve the issue of whether the ERP repetition priming effect observed in the present study reflects incidental explicit, rather than implicit, memory retrieval. Unfortunately, however, high response accuracy in the direct task (between $74 \%$ and $87 \%$ correct responses across conditions) precluded the statistical analysis of ERP to misses.
}

affect the FN400 at test when subjects had performed a verbal-conceptual encoding task at study. In the present study, we tried to constrain perceptual processing at study by using easily identifiable photographs of objects as stimuli and employing a semantic, rather than a perceptual, study task. As a consequence, we found comparable FN400 old/new effects for identical repetitions and different exemplars. Taken together, the data indicate that perceptual processing at study may be prerequisite for the emergence of FN400 perceptual specificity effects at test. Moreover, it appears that such perceptual encoding not only can be induced top-down via task instructions, but also can be triggered bottom-up as a consequence of stimulus properties.

At test, the explicit group of the present experiment performed an inclusion task, which made perceptual features task irrelevant, since both identical repetitions and different exemplars had to be accepted as old. Ecker and Zimmer (2009) recently demonstrated that the retrieval orientation adopted at test affects FN400 perceptual specificity effects. At test, they presented identical repetitions and different exemplars of studied items and found that an FN400 old/new effect for different exemplars emerged only when subjects performed an inclusion task, but not when they performed an exclusion task in which only identical repetitions had to be accepted as old. Subjects were thus able to switch flexibly between a more conceptual gist-based and a more perceptual item-specific familiarity recognition mode (see also Koutstaal, 2006). The emergence of FN400 specificity effects may, therefore, also depend critically on whether processing at retrieval is perceptually versus conceptually focused.

Recently, Voss and Paller pointed out that implicit memory processes can encroach on explicit memory tests and that the FN400 may thus reflect conceptual priming, rather than familiarity (Paller, Voss, \& Boehm, 2007; Voss, Lucas, \& Paller, 2010; Voss \& Paller, 2007). As was noted above, we do not debate that conceptual processing can contribute to familiarity-based recognition and, hence, affect the FN400 old/new effect. However, the available evidence strongly suggests that the effect cannot be purely conceptual. Apart from the already reviewed findings of perceptual specificity effects on the FN400, pervasive evidence comes from research by Stenberg and colleagues, who were able to demonstrate a clear dissociation between conceptual priming and FN400 modulation (Stenberg, Hellman, Johansson, \& Rosén, 2009; Stenberg, Johansson, Hellman, \& Rosén, 2010). In a recognition memory task using famous and unknown faces, they found that fame exclusively modulated recognition and the corresponding LPC. In contrast, frequency of names associated with the faces modulated familiarity and the FN400. At the same time, conceptual priming proved to be sensitive to fame but 
not frequency, lending strong support to the notion that the FN400 is not an index of conceptual priming.

As with the FN400, same and different objects elicited LPC old/new effects of similar magnitude. Identical repetitions did elicit a marginally more pronounced LPC that was suggestive of some differences in processing between study items and different exemplars. The perceptual mismatch was, however, not sufficient to produce a reliable effect of study-test congruency. Previous research has established a correspondence between the LPC and recollection mechanisms (e.g., Rugg \& Curran, 2007). On the premise that the LPC reflects recollection, the present result is again in line with the type-token model, which conceives recollection as a rather controlled process based on higher-level representations, so-called episodic tokens, that integrate lower-level object tokens with arbitrary contextual information (for a similar model, see Diana, Reder, Arndt, \& Park, 2006; Reder et al., 2000). As such, it should allow for a flexible reintegration of task-relevant stimulus attributes (Ecker et al., 2007a; Herron \& Rugg, 2003). The emergence of specificity effects in the LPC may thus critically depend on whether perceptual features are relevant for the memory task to be performed (e.g., Groh-Bordin et al., 2006). The inclusion task used in the present study puts little emphasis on perceptual features, since both identical and modified repetitions have to be accepted as old. In line with the present results, other studies using inclusion tasks also observed comparable LPC old/new effects for same and different stimuli (Curran \& Dien, 2003; Ecker et al., 2007a; Groh-Bordin et al., 2005; Tsivilis et al., 2001; but see Ecker \& Zimmer, 2009). In contrast, studies employing exclusion tasks in which perceptual features are made task relevantsince modified items have to be rejected as new-showed significantly more pronounced LPC old/new effects for same old items than for modified items (Curran, 2000; Curran \& Cleary, 2003; Ecker et al., 2007a; Ranganath \& Paller, 1999).

To summarize, we found not only that implicit and explicit remembering generated clearly dissociable ERP components, but also that these ERP components were differentially affected by exemplar changes from study to test. Repetition priming effects emerged only for identical repetitions, but not for different exemplars of old items, indicating that the perceptual representations accessed by implicit memory include stimulus features diagnostic for identifying single object instances. In the direct memory task, same and different exemplars elicited comparable FN400 and LPC old/new effects, indicating that both familiarity and recognition are adaptable to task demands and that the representations underlying these processes bind conceptual as well as perceptual information. As such, our data support the neurocognitive type-token model of memory (Zimmer \& Ecker, 2010).

\section{References}

Biederman, I., \& Cooper, E. E. (1992). Size invariance in visual object priming. Journal of Experimental Psychology. Human Perception and Performance, 18, 121-133.

Cave, C. B., Bost, P. R., \& Cobb, R. E. (1996). Effects of color and pattern on implicit and explicit picture memory. Journal of Experimental Psychology: Learning, Memory, and Cognition, 22, 639-653.

Cooper, L. A., Schacter, D. L., Ballesteros, S., \& Moore, C. (1992). Priming and recognition of transformed three-dimensional objects: Effects of size and reflection. Journal of Experimental Psychology: Learning, Memory, and Cognition, 18, 43-57.

Curran, T. (2000). Brain potentials of recollection and familiarity. Memory \& Cognition, 28, 923-938.

Curran, T., \& Cleary, A. M. (2003). Using ERPs to dissociate recollection from familiarity in picture recognition. Cognitive Brain Research, 15, 191-205.

Curran, T., \& Dien, J. (2003). Differentiating amodal familiarity from modality-specific memory processes: An ERP study. Psychophysiology, 40, 979-988.

Diana, R. A., Reder, L. M., Arndt, J., \& Park, H. (2006). Models of recognition: A review of arguments in favor of a dual-process account. Psychonomic Bulletin \& Review, 13, 1-21.

Ecker, U. K. H., Arend, A. M., Bergström, K., \& Zimmer, H. D. (2009). Verbal predicates foster conscious recollection but not familiarity of a task-irrelevant perceptual feature-An ERP study. Consciousness and Cognition, 18, 679-689.

Ecker, U. K. H., \& Zimmer, H. D. (2009). ERP evidence for flexible adjustment of retrieval orientation and its influence on familiarity. Journal of Cognitive Neuroscience, 21, 1907-1919.

Ecker, U. K. H., Zimmer, H. D., \& Groh-Bordin, C. (2007a). Color and context: An ERP study on intrinsic and extrinsic feature binding in episodic memory. Memory \& Cognition, 35, 14831501.

Ecker, U. K. H., Zimmer, H. D., \& Groh-Bordin, C. (2007b). The influence of object and background color manipulations on the electrophysiological indices of recognition memory. Brain Research, 1185, 221-230.

Faul, F., Erdfelder, E., Lang, A.-G., \& Buchner, A. (2007). G*Power 3: A flexible statistical power analysis program for the social, behavioral, and biomedical sciences. Behavior Research Methods, 39, 175-191.

Gratton, G., Coles, M. G. H., \& Donchin, E. (1983). A new method for off-line removal of ocular artifact. Electroencephalography and Clinical Neurophysiology, 55, 468-484.

Groh-Bordin, C., Zimmer, H. D., \& Ecker, U. K. H. (2006). Has the butcher on the bus dyed his hair? When color changes modulate ERP correlates of familiarity and recollection. NeuroImage, 32, 1879-1890.

Groh-Bordin, C., Zimmer, H. D., \& Mecklinger, A. (2005). Feature binding in perceptual priming and in episodic object recognition: Evidence from event-related brain potentials. Cognitive Brain Research, 24, 556-567.

Grove, K. L., \& Wilding, E. L. (2009). Retrieval processes supporting judgments of recency. Journal of Cognitive Neuroscience, 21, $461-473$.

Guillaume, C., Guillery-Girard, B., Chaby, L., Lebreton, K., Hugueville, L., Eustache, F., et al. (2009). The time course of repetition effects for familiar faces and objects: An ERP study. Brain Research, 1248, 149-161.

Henson, R. N., Rylands, A., Ross, E., Vuilleumeir, \& Rugg, M. D. (2004). The effect of repetition lag on electrophysiological and haemodynamic correlates of visual object priming. NeuroImage, 21, 1674-1689. 
Herron, J. E., \& Rugg, M. D. (2003). Strategic influences on recollection in the exclusion task: Electrophysiological evidence. Psychonomic Bulletin \& Review, 10, 703-710.

Horner, A. J., \& Henson, R. N. (2009). Bindings between stimuli and multiple response codes dominate long-lag repetition priming in speeded classification tasks. Journal of Experimental Psychology: Learning, Memory, and Cognition, 35, 757-779.

Koutstaal, W. (2006). Flexible remembering. Psychonomic Bulletin \& Review, 13, 84-91.

Marr, D. (1982). Vision. New York: Freeman.

McCarthy, G., \& Wood, C. C. (1985). Scalp distributions of eventrelated potentials: An ambiguity associated with analysis of variance models. Electroencephalography and Clinical Neurophysiology, 62, 203-208.

Mecklinger, A. (2000). Interfacing mind and brain: A neurocognitive model of recognition memory. Psychophysiology, 37, 1-18.

Nessler, D., Mecklinger, A., \& Penney, T. B. (2001). Event related brain potentials and illusory memories: The effects of differential encoding. Cognitive Brain Research, 10, 283-301.

Paller, K. A. (2001). Neurocognitive foundations of human memory. In D. L. Mendin (Ed.), The psychology of learning and motivation: Advances in research and theory, vol. 40 (pp. 121145). San Diego, CA: Academic Press.

Paller, K. A., Voss, J. L., \& Boehm, S. G. (2007). Validating neural correlates of familiarity. Trends in Cognitive Sciences, 11, 243250

Race, E. A., Badre, D., \& Wagner, A. D. (2010). Multiple forms of learning yield temporally distinct electrophysiological repetition effects. Cerebral Cortex, 20, 1726-1738.

Rajaram, S., \& Roediger, H. L., III. (1993). Direct comparison of four implicit memory tests. Journal of Experimental Psychology: Learning, Memory, and Cognition, 19, 765-777.

Ranganath, C., \& Paller, K. A. (1999). Frontal brain potentials during recognition are modulated by requirements to retrieve perceptual detail. Neuron, 22, 605-613.

Reder, L. M., Nhouyvanisvong, A., Schunn, C. D., Ayers, M. S., Angstadt, P., \& Hiraki, K. (2000). A mechanistic account of the mirror effect for word frequency: A computational model of remember-know judgments in a continuous recognition paradigm. Journal of Experimental Psychology: Learning, Memory, and Cognition, 26, 294-320.

Roediger, H. L., III, \& McDermott, K. B. (1993). Implicit memory in normal human subjects. In F. Boller \& J. Grafman (Eds.), Handbook of neuropsychology, vol. 8 (pp. 63-131). New York: Elsevier Science.

Roediger, H. L., III, \& Srinivas, K. (1993). Specificity of operations in perceptual priming. In P. Graf \& M. E. Masson (Eds.), Implicit memory: New directions in cognition, development and neuropsychology (pp. 17-48). Hillsdale, NJ: Erlbaum.

Rugg, M. D., \& Allan, K. (2000). Memory retrieval: An electrophysiological perspective. In M. S. Gazzaniga (Ed.), The new cognitive neurosciences (pp. 805-816). Cambridge, MA: MIT Press.

Rugg, M. D., \& Curran, T. (2007). Event-related potentials and recognition memory. Trends in Cognitive Sciences, 11, 251-257.

Rugg, M. D., Mark, R. E., Walla, P., Schloerscheidt, A. M., Birch, C. S., \& Allan, K. (1998). Dissociation of the neural correlates of implicit and explicit memory. Nature, 392, 595-598.

Rugg, M. D., \& Yonelinas, A. P. (2003). Human recognition memory: A cognitive neuroscience perspective. Trends in Cognitive Sciences, 7, 313-319.

Schacter, D. L. (1994). Priming and multiple memory systems: Perceptual mechanisms of implicit memory. In D. L. Schacter \& E. Tulving (Eds.), Memory systems 1994 (pp. 233-268). Cambridge, MA: MIT Press.

Schacter, D. L., Bowers, J., \& Booker, J. (1989). Intention, awareness, and implicit memory: The retrieval intentionality criterion. In S.
Lewandowsky, J. M. Dunn, \& K. Kirsner (Eds.), Implicit memory: Theoretical issues (pp. 47-69). Hillsdale, NJ: Erlbaum.

Schacter, D. L., \& Buckner, R. L. (1998). Priming and the brain. Neuron, 20, 185-195.

Schacter, D. L., Dobbins, I. G., \& Schnyer, D. M. (2004). Specificity of priming: A cognitive neuroscience perspective. Nature Reviews Neuroscience, 5, 853-862.

Schendan, H. E., \& Kutas, M. (2003). Time course of processes and representations supporting visual object identification and memory. Journal of Cognitive Neuroscience, 15, 111-135.

Schendan, H. E., \& Kutas, M. (2007). Neurophysiological evidence for the time course of activation of global shape, part, and local contour representations during visual object categorization and memory. Journal of Cognitive Neuroscience, 19, 743-749.

Schloerscheidt, A. M., \& Rugg, M. D. (2004). The impact of change in stimulus format on the electrophysiological indices of recognition. Neuropsychologia, 42, 451-466.

Snodgrass, J. G., \& Corwin, J. (1988). Pragmatics of measuring recognition memory: Applications to dementia and amnesia. Journal of Experimental Psychology. General, 117, 34-50.

Squire, L. R. (1994). Declarative and nondeclarative memory in humans and animals: Experimental analysis and historical origins. In D. L. Schacter \& E. Tulving (Eds.), Memory systems 1994 (pp. 203-232). Cambridge, MA: MIT Press.

Srinivas, K. (1996). Contrast and illumination effects on implicit and explicit measures of memory. Journal of Experimental Psychology: Learning, Memory, and Cognition, 22, 1123-1135.

Stenberg, G., Hellman, J., Johansson, M., \& Rosén, I. (2009). Familiarity or conceptual priming: Event-related potentials in name recognition. Journal of Cognitive Neuroscience, 21, 447460.

Stenberg, G., Johansson, M., Hellman, J., \& Rosén, I. (2010). Do you see yonder cloud?"-On priming concepts, a new test, and a familiar outcome. Reply to Lucas et al:: "Familiarity or conceptual priming? Good question! Comment on Stenberg, Hellman, Johansson, and Rosén (2009). Journal of Cognitive Neuroscience, 22, 618-620.

Treisman, A. (2006). Object tokens, binding and visual memory. In H. D. Zimmer, A. Mecklinger, \& U. Lindenberger (Eds.), Handbook of binding and memory: Perspectives from cognitive neuroscience (pp. 315-338). Oxford: Oxford University Press.

Treisman, A., \& Gelade, G. (1980). A feature-integration theory of attention. Cognitive Psychology, 12, 97-136.

Tsivilis, D., Otten, L. J., \& Rugg, M. D. (2001). Context effects on the neural correlates of recognition memory: An electrophysiological study. Neuron, 31, 497-505.

Tulving, E., \& Schacter, D. L. (1990). Priming and human memory systems. Science, 247, 301-306.

Urbach, T. P., \& Kutas, M. (2002). The intractability of scaling scalp distributions to infer neuroelectric sources. Psychophysiology, 39, 791-808.

Vogel, E. K., Luck, S. J., \& Shapiro, K. L. (1998). Electrophysiological evidence for a postperceptual locus of suppression during the attentional blink. Journal of Experimental Psychology. Human Perception and Performance, 24, 1656-1674.

Voss, J. L., Lucas, H. D., \& Paller, K. A. (2010). Conceptual priming and familiarity: Different expressions of memory during recognition testing with distinct neurophysiological correlates. Journal of Cognitive Neuroscience, 22, 2638-2651.

Voss, J. L., \& Paller, K. A. (2007). Neural correlates of conceptual implicit memory and their contamination of putative neural correlates of explicit memory. Learning \& Memory, 14, 259-267.

Weldon, M. S., Roediger, H. L., III, Beitel, D. A., \& Johnston, T. R. (1995). Perceptual and conceptual processes in implicit and explicit tests with picture fragment and word fragment cues. Journal of Memory and Language, 34, 268-285. 
Wilding, E. L. (2006). The practice of rescaling scalp-recorded eventrelated potentials. Biological Psychology, 72, 325-332.

Yonelinas, A. P. (2002). The nature of recollection and familiarity: A review of 30 years of research. Journal of Memory and Language, 46, 441-517.

Zimmer, H. D. (1995). Size and orientation of objects in explicit and implicit memory: A reversal of the dissociation between perceptual similarity and type of test. Psychological Research, 57, 260-273.

Zimmer, H. D., \& Ecker, U. K. H. (2010). Remembering perceptual features unequally bound in object and episodic tokens: Neural mechanisms and their electrophysiological correlates. Neuroscience and Biobehavioral Reviews, 34, 1066-1079.

\section{Author notes}

Reprint requests should be sent to Kristina Küper (kristina.kueper@mx.uni-saarland.de). This article is based on a reanalysis of data that were collected and first analyzed by Groh-Bordin as part of his dissertation project. This research was supported by the German Research Foundation (Deutsche Forschungsgemeinschaft, Grant FOR448-Zi 308/4-2 to H. D. Zimmer). We thank Deanna Barch and three anonymous reviewers for their helpful comments on a previous version of the manuscript. 